\title{
Mechanisms behind slow photo-response character of Pulsed Electron Deposited ZnO thin films
}

\author{
Mehmet Özdoğan ${ }^{1,2}$, Gökhan Utlu ${ }^{1}$, and Cem Çelebi ${ }^{2 *}$ \\ ${ }^{1}$ Department of Physics, Faculty of Science, Ege University, 35100, Izmir, Turkey \\ ${ }^{2}$ Quantum Device Laboratory, Department of Physics, Izmir Institute of Technology, 35430, \\ Izmir, Turkey \\ *e-mail address: cemcelebi@iyte.edu.tr
}

\begin{abstract}
Zinc Oxide ( $\mathrm{ZnO})$ semiconductor is ideal candidates for ultra-violet (UV) photodetector due to its promising optoelectronic properties. Photodetectors based on $\mathrm{ZnO}$ nanostructures show very high photoconductivity under UV light, but they are plagued by slow photo-response time as slow as several tens of hours, even more. Most of the studies claimed that atmospheric adsorbates such as water and oxygen create charge traps states on the surface and remarkably increase both the photoconductivity and response time, but there are also limited studies that claiming the defect induced states acting as hole trap centers responsible for these problems. However, the underlying physical mechanism is still unclear. Here we study the effects of both adsorbates and defect-related states on the photo-response character of Pulsed Electron Deposited ZnO thin films. In order to distinguish between these two mechanisms, we have compared the time-dependent photo-response measurements of bare-ZnO and $\mathrm{SiO}_{2}$ encapsulated-ZnO thin film samples taken under $\mathrm{UV}$ light and high vacuum. We show that the dominant mechanism of photo-response in $\mathrm{ZnO}$ is the adsorption/desorption of oxygen and water molecules even when the measurement is performed in high vacuum. When the samples are encapsulated by a thin $\mathrm{SiO}_{2}$ layer, the adsorption/desorption rates can significantly improve, and the effects of these molecules partially removed.
\end{abstract}

Keywords: Zinc Oxide, Pulsed Electron Deposition, photo-response, $\mathrm{SiO}_{2}$, encapsulation, defects, atmospheric adsorbates.

\section{Introduction}

Zinc Oxide $(\mathrm{ZnO})$ material has received considerable attention due to its direct wide bandgap (3.37 eV) and high exciton binding energy (60 meV) for optoelectronic applications such as ultra-violet (UV) light detection for a long time [1]. Numerous studies have been conducted to achieve realization of electrically stable nano-electronic and nano-optoelectronic 
devices based on $\mathrm{ZnO}$ that are expected to operate a long time with reduced noise levels under atmospheric conditions; however, the electrical and optoelectronic properties of $\mathrm{ZnO}$ material are often changed over time [2,3]. Nanostructured or thin film forms of $\mathrm{ZnO}$ material are highly sensitive to environment because of their surface defects serving adsorption sites for atmospheric gases on the surface [4], which can be utilized sensitive gas detection performance [5]. Reactive gases like $\mathrm{O}_{2}$ and $\mathrm{H}_{2} \mathrm{O}$ in ambient can act as electron trap centers when they are stuck on the surface of n-type materials such as $\mathrm{ZnO}$. Unintentional adsorption/desorption of these molecules can randomly modify the electronic transport characteristics of these kinds of materials [6-9]. The electrical conductivity of n-type $\mathrm{ZnO}$ material decreases upon the adsorption of these gas molecules since the main charge carriers (electrons) are captured, or increases upon desorption of these molecules from the surface $[10,11]$. It has been shown that the adsorbates like $\mathrm{O}_{2}$ and $\mathrm{H}_{2} \mathrm{O}$ can significantly change the carrier density of $\mathrm{ZnO}$ and strongly dominate the photo-response characteristics of UV sensitive photodetectors fabricated out of $\mathrm{ZnO}$ thin films or other forms [12]. Therefore, it is of great importance to unveil the detailed physical mechanism underlying adsorbate induced electrical changes prior to designing solid-state devices out of $\mathrm{ZnO}$ n-type semiconductor.

In $\mathrm{ZnO}$ materials, high photoconductivity and slow photo-responsivity characters to the UV light have been simultaneously observed not only due to their high sensitivity to atmosphere [13] but also their intrinsic defects acting as hole trap centers within the bandgap that prolong their response time [14]. Most of the studies suggested that the slow photoresponse character of $\mathrm{ZnO}$ originates from atmospheric adsorbates such as oxygen and water molecules that create charge trap states on the surface. For example, Ahn et al. [15] proposed that the slow photo-response in sol-gel synthesized $\mathrm{ZnO}$ nanowires is due to the charge transfer between water molecules and $\mathrm{ZnO}$ nanowire. Also, Soci et al. [6] obtained very high photoconductive gain as high as $\mathrm{G} \sim 10^{8}$ for $\mathrm{ZnO}$ nanowire by means of desorption of oxygen. Moreover, Li et al. [10] proposed that the reason is due to the both effects of oxygen and water molecules. On the other hand, there are also limited studies that claiming the suspects are defective states behaving hole trap centers that prolong the response time. For instance, Moazzami et al. [14] reported that the photo-response of $\mathrm{ZnO}$ epilayers was dominated by shallow and deep trap states. However, the reason of higher but slower photo-response character of $\mathrm{ZnO}$ cannot be explained via only adsorption/desorption of atmospheric species $\left(\mathrm{O}_{2}\right.$ and/or $\left.\mathrm{H}_{2} \mathrm{O}\right)$ or defect-induced levels within the bandgap. It should be due to combination of both effects. In previous work [16], we have shown that in high vacuum, the photo- 
response was bigger and slower than that in air, confirming the atmospheric adsorbates such as $\mathrm{O}_{2}$ and $\mathrm{H}_{2} \mathrm{O}$ greatly dominate the photo-responsivity. And, they suppress the effects of defect states. In this work, therefore, we have developed an experimental approach to distinguish the effect of defect-states acting as hole trap centers from the effect of atmospheric adsorbates by simply encapsulation of $\mathrm{ZnO}$ thin film surface with a thin $\mathrm{SiO}_{2}$ layer. We show that encapsulation of $\mathrm{ZnO}$ thin films with $\mathrm{SiO}_{2}$ partially removes the contribution of adsorbates to photo-response with a substantial reduction of adsorption and desorption times.

The objective of this work is to explain the aforementioned mechanisms more comprehensively and systematically, thus this study contributes to a better understanding of the ambient and intrinsic defects effects on the slow photo-response character of $\mathrm{ZnO}$ nanostructures than previously reported works, and helps guide the design of ZnO-based photodetectors or gas sensors. Additionally, many encapsulation approaches have been employed for 2D materials such as $\mathrm{HfO}_{2}$ [17] and PMMA [18] for $\mathrm{MoS}_{2}, \mathrm{SiO}_{2}$ for graphene [19]; yet, there is no detailed study investigating encapsulation effect on the photo-response properties of $\mathrm{ZnO}$ thin film.

\section{Experimental Details}

Before the deposition of $\mathrm{ZnO}$ thin films, $\mathrm{Cr}(3 \mathrm{~nm}) / \mathrm{Au}(80 \mathrm{~nm})$ source/drain electrodes were patterned on $10 \mathrm{~mm}$ x $10 \mathrm{~mm}$ fused-quartz substrates by thermal evaporation technique, and the inset of Fig. 1(b) shows a typical device schematic. The channel length between source and drain electrodes was set to $200 \mu \mathrm{m}$, and this configuration was used to Transient Photocurrent Spectroscopy (TPS) measurements. Employing a shadow mask, $185 \mathrm{~nm}$ thick ZnO films with a size of $4 \mathrm{~mm} \times 4 \mathrm{~mm}$ were deposited at the center of the substrates by Pulsed Electron Deposition (PED) method. The details of PED technique can be found in Ref. [20]. The deposition parameters were fine-tuned to achieve the best ablation and plume (i.e., plasma and evaporated material) intensity, and to get high crystal quality of $\mathrm{ZnO}$ films. The deposition of $\mathrm{ZnO}$ thin films were done with a substrate temperature of $400^{\circ} \mathrm{C}$, an oxygen pressure of $1.57 \mathrm{~Pa}$, an electron discharge voltage of $15 \mathrm{kV}$, and a pulse frequency of $5 \mathrm{~Hz}$. Then, on the top of $\mathrm{ZnO}$ thin film, a thin layer of $\mathrm{SiO}_{2}(\sim 200 \mathrm{~nm}$ thick and $5 \mathrm{~mm}$ x $5 \mathrm{~mm}$ sized) was deposited using PED system as an encapsulation layer to assure full coverage of surface. For comparison, a set of bare $\mathrm{ZnO}$ thin films were also deposited by using the same protocols mentioned above. 
After the successful fabrication of $\mathrm{ZnO}$ thin films, the structural, electrical, and optoelectronic properties of samples have been evaluated by different characterization techniques. Scanning Electron Microscopy (SEM) was performed using Quanta 250 instrument with typically operated at $5.0 \mathrm{kV}$ to characterize the $\mathrm{ZnO}$ thin film morphologies.

The XPS analyses were performed using a Thermo Scientific Model K-Alpha XPS instrument with monochromatic $\mathrm{Al} \mathrm{K \alpha}$ radiation $(1486.68 \mathrm{eV})$ under operating pressure set to $2 \times 10^{-7} \mathrm{~Pa}$. The survey spectrum scan was completed by taking the average of 10 scans with X-ray spot size at $300 \mu \mathrm{m}$ and passing energy at $30 \mathrm{eV}$. All the XPS spectra were calibrated by taking the C $1 \mathrm{~s}$ peak located at $\sim 284 \mathrm{eV}$ as a reference. Data were analyzed using Avantage XPS software package. Peak fitting was done using Shirley/Smart type background and Gaussian/Lorentzian convolution shapes.

The X-ray diffraction (XRD) was carried out via a Philips X'Pert Pro Theta/2Theta Diffractometer with a copper K-Alpha X-ray source $(\lambda=1.540 \AA)$. The scans were performed from $20^{\circ}$ to $80^{\circ}$ in $0.001^{\circ}$ steps.

The Photoluminescence (PL) spectra of the samples were taken by a Perkin Elmer LS55 Luminescence Spectrometer (with a pulsed Xenon lamp) at an excitation wavelength of $350 \mathrm{~nm}$ with both $10 \mathrm{~nm}$ excitation and emission slit widths at room temperature. The excitation energy of $3.57 \mathrm{eV}$ (350 nm) was slightly above the $3.26 \mathrm{eV}$ typical bandgap of our PED-fabricated samples.

Electrical and opto-electronic characterizations of the samples were done under 254 $\mathrm{nm}$ wavelength UV light illumination (output power of $3 \mathrm{~mW}$ ) inside a high vacuum chamber with a base pressure of about $4 \times 10^{-3} \mathrm{~Pa}$. For TPS experiments, an electronic shutter mechanism is coupled to the UV light source. The photocurrent data of the samples were acquired by using Keithley 6485 Picoammeter, and Keithley 2400 Source Meter. During measurement, the applied voltage between source and drain was kept constant at $0.5 \mathrm{~V}$. Prior to the each set of measurements, we conducted I-V measurements for bare and encapsulated $\mathrm{ZnO}$ thin films (denoted as $\mathrm{B}-\mathrm{ZnO}$ and $\mathrm{E}-\mathrm{ZnO}$, respectively) before and after TPS measurements, and then the samples were left overnight under ambient conditions in order to return back to their initial states. Afterwards, the samples were exposed to UV light for about $3 \mathrm{~h}$. in high vacuum to remove existing adsorbates from the surface. Right after $3 \mathrm{~h}$ of continuous UV light exposure, TPS measurements were conducted for short periods as $30 \mathrm{~s}$ 
with three on/off cycles to reveal time dependent photo-response characteristics of the samples.

\section{Results}

The SEM image of ZnO thin film exhibits nanoparticles appearing as white spots on the film surface as seen in Fig. 1(a). These particles are more likely to occur as a result of thin film fabrication with high energy deposition techniques such as Pulsed Laser Deposition (PLD) and PED [21,22]. However, the origin of such nanoparticles is a matter of discussion. They can be directly emitted from target during ablation process, or they can be occurred in the gas phase, during transfer of species from the target toward the substrate [23]. In addition, we conducted EDX analysis to identify the elemental compositions of these nanoparticles, and the results showed that they possess almost same compositions of other regions on the film surface. The presence of such particles on the film surface increases the surface area-tovolume ratio, which maximizes the interaction between surface and atmospheric gases, and makes it more sensitive to the environment.
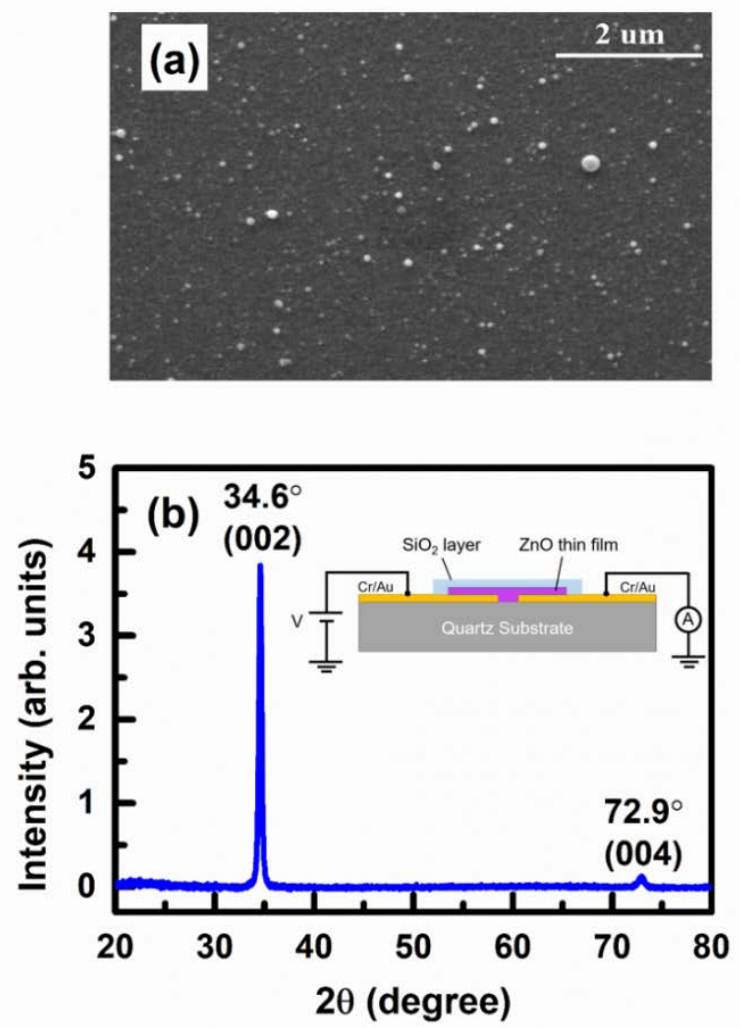

Fig. 1. (a) SEM image and (b) XRD pattern of bare ZnO thin film. The inset of (b) shows typical device schematic of $\mathrm{SiO}_{2}$ encapsulated $\mathrm{ZnO}$ device. 
Fig. 1(b) displays the XRD pattern of B-ZnO thin film. As seen in XRD graph, the PED-fabricated $\mathrm{ZnO}$ thin films have a single sharp peak at an angle of around $34.6^{\circ}$ and very small peak around $72.9^{\circ}$ which were identified as (002) and (004) orientations of hexagonal wurtzite $\mathrm{ZnO}$ crystal structure. It indicates that the $\mathrm{ZnO}$ thin film was deposited along a c-axis orientation of the quartz substrate. This c-axis orientation of crystallites is classically observed for $\mathrm{ZnO}$ films, whatever the method of deposition and the substrate nature [20]. The average crystallite size (D) of ZnO thin films was calculated using the (002) major diffraction peak by Debye-Sherrer formula $(D=0.89 \lambda / \beta \cos \theta)$, and it was found to be $30 \mathrm{~nm}$. This value is comparable to that of PLD-fabricated $\mathrm{ZnO}$ thin films [24].
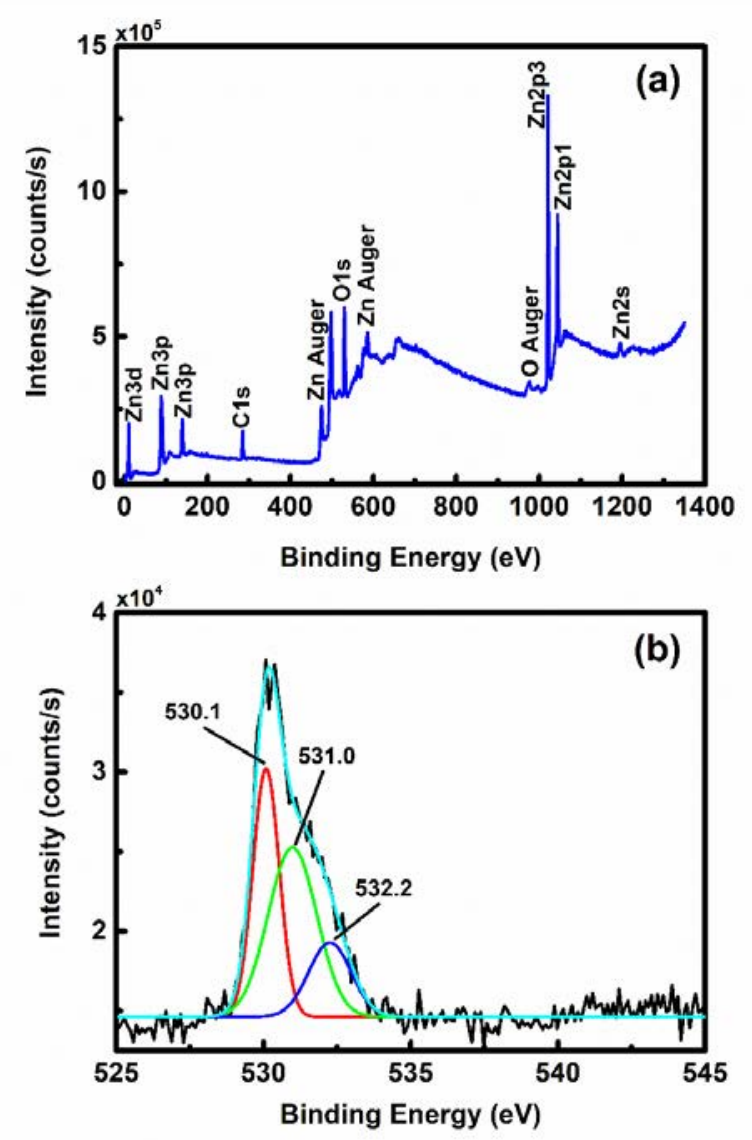

Fig. 2. The XPS spectra of the PED-fabricated bare $\mathrm{ZnO}$ thin film. A full range survey spectrum was shown in (a), while high-resolution XPS chemical binding spectra O 1 s state (its deconvolution) was shown in (b).

XPS analysis was performed to get information about the surface-adsorbed species and surface chemical states of the PED-fabricated ZnO thin films. Fig. 2 (a) shows XPS survey spectra, and no elements besides $\mathrm{Zn}, \mathrm{O}$ and $\mathrm{C}$ were detected. The presence of Zn2p3/2 and Zn2p1/2 peaks locating at $1021.3 \mathrm{eV}$ and $1044.7 \mathrm{eV}$ verify that $\mathrm{Zn}$ exists in oxidized states. The energy difference between two $\mathrm{Zn}$ components is $23.4 \mathrm{eV}$, which is agreement with the 
reported values of $\mathrm{ZnO}$ [4]. Fig. 2(b) shows the high-resolution XPS spectra of O1s core level. In order to further examination, the O1s peak was deconvoluted by fitting with three Gaussian/Lorentzian peaks. The deconvoluted peaks are located at binding energies 530.1, 531.0 and $532.2 \mathrm{eV}$. Here, the lower energy peak is attributed to lattice oxygen in hexagonal wurtzite structure of $\mathrm{ZnO}$ [25-27]. The middle peak at binding energy of $531.0 \mathrm{eV}$ is associated with oxygen vacancy in $\mathrm{ZnO}$ lattice which confirms the existence of surface defects on $\mathrm{ZnO}$ thin film. The higher energy peak corresponds to chemisorbed oxygen species such as $\mathrm{OH}^{-}, \mathrm{H}_{2} \mathrm{O}$ and $\mathrm{O}_{2}[4,28,29]$.

The PL measurements were performed using a pulsed Xenon lamp with an excitation wavelength of $350 \mathrm{~nm}$ at room temp. to unveil the defect states in the PED-fabricated $\mathrm{ZnO}$ thin films, and Fig. 3(a) shows the PL spectrum. The raw PL data were processed using baseline subtraction and $2^{\text {nd }}$ order Savitzky-Golay smoothing, and it was deconvoluted by fitting nine Gaussian peaks to reveal hidden peaks. As seen in Fig. 3(a), the PL graph shows near-band emission at an energy of $3.17 \mathrm{eV}(391.4 \mathrm{~nm})$. Besides the near-band emission, the PED-fabricated ZnO thin films show visible emissions in the spectrum region $2.94-2.18 \mathrm{eV}$ consisting of several distinct peaks at $2.94 \mathrm{eV}(421.4 \mathrm{~nm}), 2.79 \mathrm{eV}(445.2 \mathrm{~nm}), 2.71 \mathrm{eV}$ (457.3 nm), $2.55 \mathrm{eV}$ (486.6 nm), $2.39 \mathrm{eV}$ (518.5 nm), $2.35 \mathrm{eV}$ (528.4 nm), $2.29 \mathrm{eV}$ (541.3 $\mathrm{nm})$, and $2.18 \mathrm{eV}(568.4 \mathrm{~nm})$. Basically, transition from valance band (VB) to conduction band (CB), and VB to shallow defect levels occurs upon photo-excitation in the PL measurement, which give rise to subsequent emissions; from CB to deep levels, shallow levels to VB, shallow levels to deep levels, and hole capture at deep levels gives violet, blue and green emissions according to energy levels difference [29]. Since the excitation energy (3.57 eV) was slightly above the energy bandgap of our samples $(\sim 3.26 \mathrm{eV}$ reported in our previous work [16]); therefore, the electrons excited from the VB to the CB as well as shallow defect levels.

Generally oxygen vacancies are considered as the origin of observed green emission in the PL spectrum. On the other hand, zinc related defects such as; zinc interstitials (neutral, single and double ionized) are responsible for blue emission. According to the previous reports [2934], the observed emissions in PL spectrum (Fig. 3(a)) can be summarized as follows: the transitions from 1) excitonic states to $\mathrm{VB}$, or zinc interstitial levels to $\mathrm{VB}, 2) \mathrm{CB}$ to $\mathrm{O}$ interstitials levels, 3) double or single ionized Zn vacancy levels to VB, 4) double ionized Zn interstitials levels to VB, 5) single ionized Zn interstitials levels to neutral Zn vacancy levels, 6) single ionized $\mathrm{O}$ vacancy levels to $\mathrm{VB}, 7$ ) $\mathrm{CB}$ to neutral antisite $\mathrm{O}$ levels, 8) $\mathrm{CB}$ to antisite 
O or interstitials O levels, and 9) CB to double ionized O vacancy levels. The first four peaks (2-5) are responsible for blue emission, whereas last four peaks (6-9) are responsible for green emission. The numbers correspond to those marked peaks in Fig. 3(a).

The observed possible transitions in the PL spectrum evidence that the PED-fabricated $\mathrm{ZnO}$ thin films possess many defects levels within the bandgap which are induced by $\mathrm{O}$ and $\mathrm{Zn}$ vacancies, $\mathrm{O}$ and $\mathrm{Zn}$ interstitial atoms, and antisite $\mathrm{O}$ atoms. These defects states were unintentionally introduced the $\mathrm{ZnO}$ structure during PED ablation process. These defectrelated states also give rise to visible-light response ability in addition to the intrinsic UV light detection capability of $\mathrm{ZnO}$ as reported in Refs. [14,29].
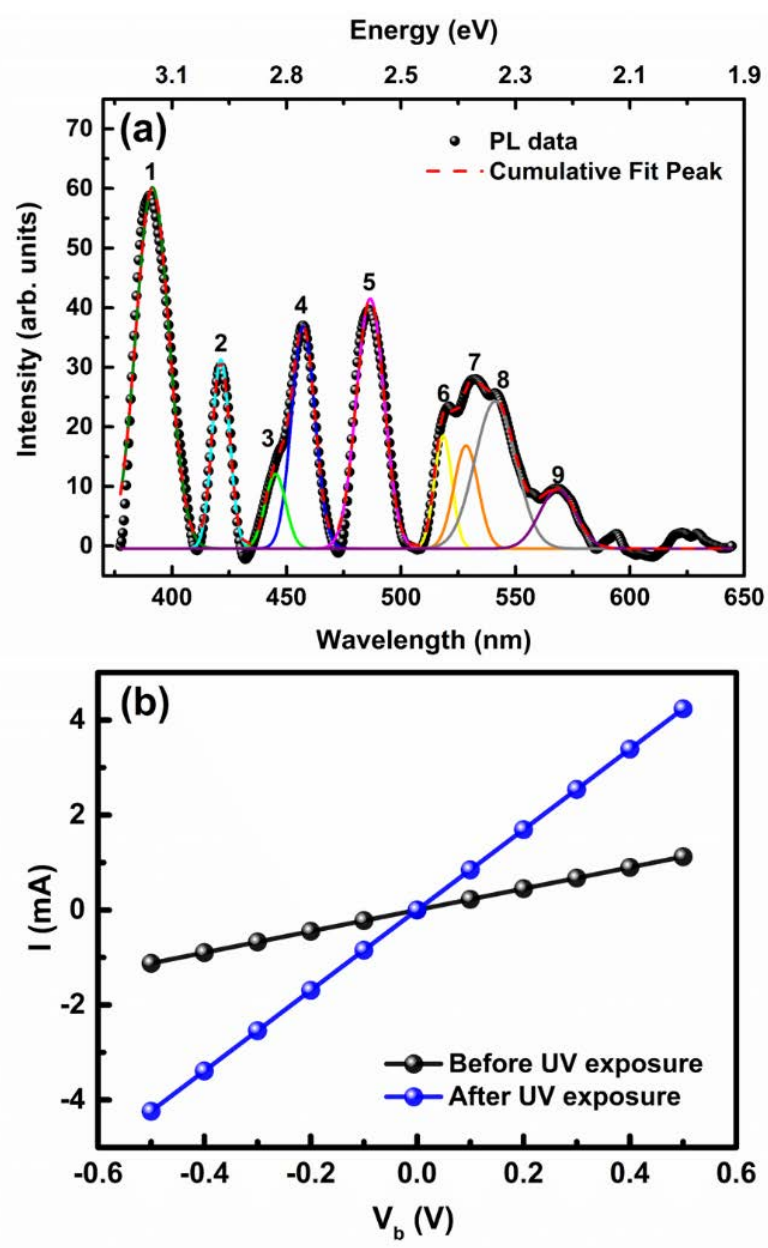

Fig. 3. (a) PL spectrum of $\mathrm{B}-\mathrm{ZnO}$ thin film. In here, black circles correspond to the smoothed and baseline subtracted PL data. The numbers correspond to the deconvoluted peaks (b) I-V measurements of B-ZnO sample before and after $3 \mathrm{~h}$ of UV light exposure. The current data are acquired by sweeping voltage in $0.2 \mathrm{~V}$ steps.

Fig. 3(b) demonstrates the I-V characteristics of B-ZnO thin film device before and after UV light exposure. It was observed that the I-V curves were perfectly linear and symmetric in both bias regions (forward and reverse), indicating good ohmic contact nature. 
This means that the device, which was fabricated as metal-semiconductor-metal, operates as photo-conductor. The initial resistance of $\mathrm{ZnO}$ thin film was determined from the slope of I-V curve as $0.45 \mathrm{k} \Omega$. After $3 \mathrm{~h}$ of UV light illumination under high vacuum, the resistance of $\mathrm{ZnO}$ thin film reduced to $0.12 \mathrm{k} \Omega$. The observed drop in the resistance can be explained as in the following manner. The atmospheric molecules such as $\mathrm{O}_{2}$ and $\mathrm{H}_{2} \mathrm{O}$ are readily adsorbed onto surface of sample after exposure to air. These adsorbates behave like electron trapping surface states, and hence reduce the electron density of n-type $\mathrm{ZnO}$ material. As the UV illumination causes desorption of these adsorbates from the sample surface into vacuum, the trapped electrons are released back to the structure. Therefore, the resistance of the UVilluminated sample becomes lower than that of as-grown $\mathrm{ZnO}$ thin film.

Now we turn to time-dependent photo-response measurements. All measurements were done in a high vacuum (low $10^{-3} \mathrm{~Pa}$ ) to ensure a controllable environment. During these measurements, the applied voltage between source and drain was kept constant at $0.5 \mathrm{~V}$. To promote desorption of adsorbates which were already adsorbed on the surface in air, the samples were exposed to UV light. The UV irradiation wavelength was specifically selected to be $254 \mathrm{~nm}(\sim 4.9 \mathrm{eV})$ since it is energetically sufficient enough to remove chemisorbed $\mathrm{O}_{2}$ and $\mathrm{H}_{2} \mathrm{O}$ molecules on the surface [19] as well as for generating electron-hole pairs in the depletion regions of $\mathrm{ZnO}$ thin film ( $\mathrm{E}_{\mathrm{g}} \sim 3.26 \mathrm{eV}$ [16]). Time-dependent current variation of $\mathrm{B}-\mathrm{ZnO}$ thin film photo-conductor was shown in Fig. 4(a). The variation in the measured current $\Delta I / I_{0}(\%)$ were determined by using the following expression;

$$
\left(\frac{\Delta I}{I_{0}}\right) \times 100 \%=\left(\frac{I-I_{0}}{I_{0}}\right) \times 100 \%
$$

where $I_{0}$ is the dark current read before UV exposure and $I$ is the measured current after UV exposure. Upon the UV light exposure the photo-response of sample initially increased rapidly, and a subsequent slower exponential growth was observed. After the UV light source was turned off, fast and slow exponential decay trends were observed similarly to the exponential growing parts. After 5 ks of UV illumination, the time necessary to reach a stable high vacuum condition in vacuum probe station, the variation in current of $\mathrm{B}-\mathrm{ZnO}$ sample was measured as high as $\sim 350 \%$. This result supports the high photoconductive gain of $\mathrm{ZnO}$ previously mentioned in many reports [6]. After turning the UV light off, the current variation didn't decrease initial level (dark level) since the oxygen and water molecules desorbed by the UV light were pumped away, and thus, the concentration of adsorbate molecules re-adsorbed on the surface is smaller than it was prior to illumination. 

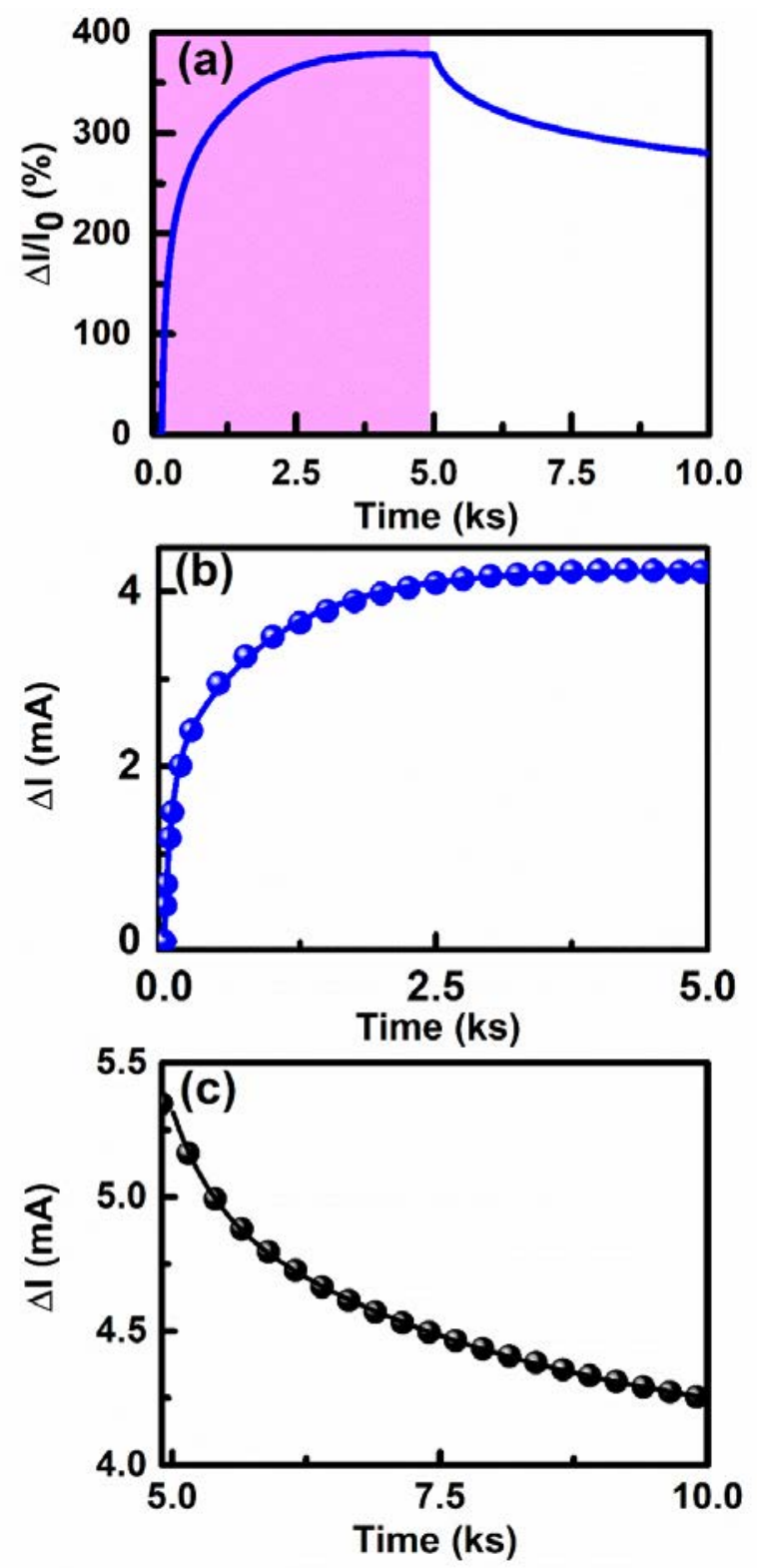

Fig. 4. (a) The change in the currents of $\mathrm{B}-\mathrm{ZnO}$ thin film that was exposed to $254 \mathrm{~nm}$ UV light for a period of 5 ks under high vacuum. After a period of $5 \mathrm{ks}$, the UV light was turned off and the sample was let to relax in high vacuum for another $5 \mathrm{ks}$. The violet region shows the time interval when the UV light is on. $\Delta I=I-I_{0}(m A)$, where $I_{0}$ denotes the current read before UV illumination and $I$ is the current after UV illumination. During measurement, the applied voltage between source and drain was kept constant at $0.5 \mathrm{~V}$. The growing part of photo-response variation of (a) B-ZnO thin film was shown in (b) with blue spheres, while the decaying part was shown in (c) with black spheres. Blue and red curves illustrate the fitted curves using Eq. (1) and (2), respectively.

As mentioned above, the photo-response characteristics of $\mathrm{ZnO}$ exhibit an exponential raise and decay behaviors determined by the UV light is turned on-off. The photo-response raised exponentially upon UV light exposure was fitted by the sum of two-exponential growth functions; 


$$
I=I_{0}+A_{1}\left(1-\exp \left(-t / \tau_{f}\right)\right)+A_{2}\left(1-\exp \left(-t / \tau_{s}\right)\right)
$$

and it was shown in Fig. 4(b) with a blue line. In equation (2), $A_{1}$ and $A_{2}$ fit constants, $I_{0}$ correspond to related dark current read before the illumination, $I_{0}=4.27 \mathrm{~mA}$ (ohmic contact leads to high dark current), and the extracted time constants were $\tau_{f}=0.09 \mathrm{ks}$ and $\tau_{s}=0.97 \mathrm{ks}$ corresponding to the first and second terms of the Eq. (2), respectively. Here $\tau_{f}$ is the time constant of fast component and $\tau_{s}$ is the time constant of slow component. When turning the UV light off, the observed decrease in photo-response was fitted by the sum of twoexponential decay functions as;

$$
I=I_{0}+A_{1} \exp \left(-t / \tau_{f}\right)+A_{2} \exp \left(-t / \tau_{s}\right)
$$

where $I_{0}=4.06 \mathrm{~mA}$, and the extracted time constants are $\tau_{f}=0.36 \mathrm{ks}$ and $\tau_{s}=3.14 \mathrm{ks}$ corresponding to the first and second terms in the Eq. (3), respectively. The fitted curve was shown in Fig. 4(c) with a black line.

In our previous work [16], we have compared the photo-response properties of PEDfabricated $\mathrm{ZnO}$ thin film for air and high vacuum environments, and we saw that the photoresponse decreases faster in air (with a \%45 relative humidity) and reaches initial condition upon turning the light off. When the water molecules are adsorbed with the hydrogen sides on the surface, which are positively charged due to the high electronegativity of oxygen than hydrogen [15], they capture more electrons than oxygen. In addition, Li et al. [10] reported that water adsorption especially at high humidity cases (> 70\%) leads to the fast shortening of the photocurrent in decaying stage for $\mathrm{ZnO}$ nanowires. Moreover, Panda et al. [35] reported two time constants for current decaying stage of thermally grown $\mathrm{ZnO}$ thin films, and they related the fast decay time to water adsorption, the slow decay rate to oxygen adsorption. According to our results and literature, we concluded that the estimated two fast time constants in above are more likely to occur with photo-assisted desorption/adsorption of $\mathrm{H}_{2} \mathrm{O}$ molecules from the surface, whereas the slow time constants are due to relatively slow desorption/adsorption rate of $\mathrm{O}_{2}$ molecules from the surface.

As seen in Fig. 4(a), the growing and decaying part of photo-response variation are not symmetric. This clearly shows the effect of atmospheric adsorbates on the photo-response characteristics of our samples. In the decaying part, the fast time constant is 4 times as large relative to fast time constant for growing part (0.36 ks / $0.09 \mathrm{ks})$ corresponding to readsorption of $\mathrm{H}_{2} \mathrm{O}$, whereas the slow time constant is $\sim 3.2$ times higher than that of the growing part (3.14 ks $/ 0.97 \mathrm{ks}$ ) as a consequence of re-adsorption of $\mathrm{O}_{2}$ molecules on the 
surface. When the high vacuum reached its stable value $\left(\sim 5.0 \times 10^{-3} \mathrm{~Pa}\right)$, the desorbed $\mathrm{H}_{2} \mathrm{O}$ and $\mathrm{O}_{2}$ molecules from the surface with the help of UV light were mostly evacuated from the environment. Therefore, fast and slow time constants were largely increased since readsorption rates were greatly decreased due to lack of adsorbate molecules, and consequently an asymmetric trend was observed in the photo-response character determined by the UV light was turned on-off. This indicates that the dominant mechanism of photo-response in $\mathrm{ZnO}$ is adsorption/desorption of atmospheric species on the surface, and it is difficult to distinguish photo-generation/recombination times from these large time constants. Therefore, to eliminate adsorbate effects on the photo-response, we have encapsulated the surface of $\mathrm{ZnO}$ with a thin layer of $\mathrm{SiO}_{2}$.

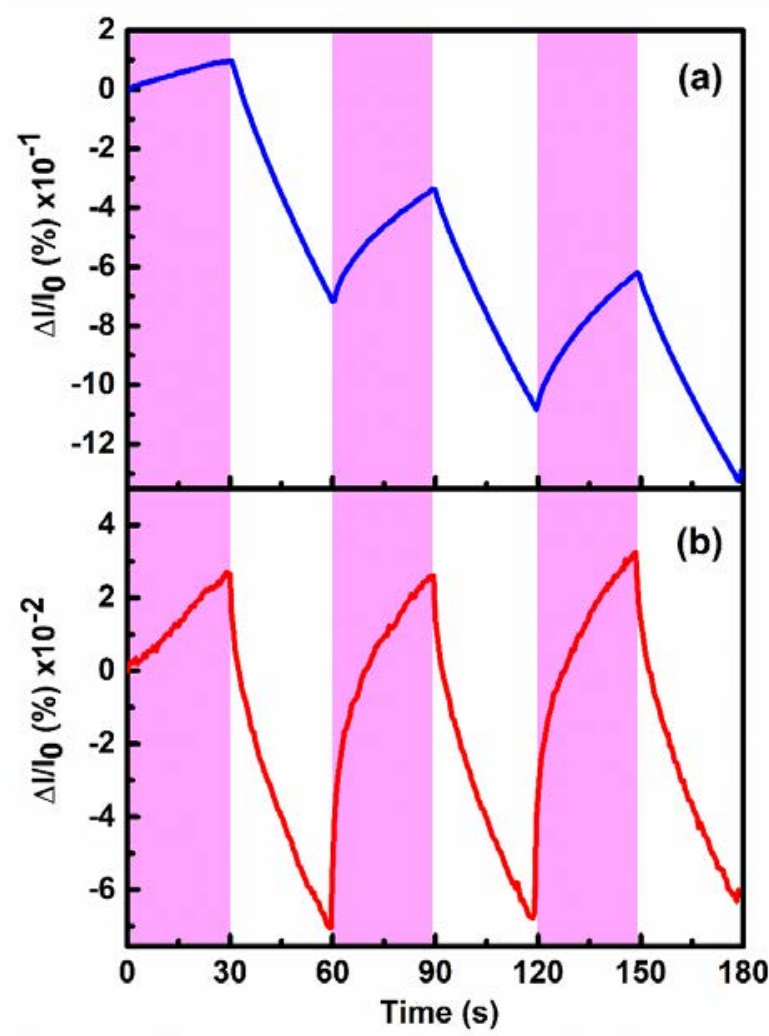

Fig. 5. Short-period TPS measurements of (a) B-ZnO thin film and (b) E-ZnO thin film. The violet regions show the time intervals when the UV light is on. During measurement, the applied voltage between source and drain was kept constant at $0.5 \mathrm{~V}$.

In Fig. 5, we have compared the photo-response behaviors of $\mathrm{B}-\mathrm{ZnO}$ and $\mathrm{E}-\mathrm{ZnO}$ thin films by taking short periods TPS measurements. The TPS measurements were conducted for short periods as $30 \mathrm{~s}$ with three on/off cycles. Prior to the these measurements, both samples were exposed to UV light in high vacuum for duration of $\sim 3$ h to make sure that all possible adsorbates were removed greatly from surfaces. To quantitatively examine the results, short- 
period TPS curves were fitted using the same two-component exponential functions presented above in Eqs. (2) and (3), and thus the time constants were extracted. For the experiments, we first started to short period TPS measurements with UV light was on state, and after $30 \mathrm{~s}$ time interval, the shutter was closed (off state). In Fig. 5(a), following a very small but sharp drop within $3.2 \mathrm{~s}$, an exponential decay trend in the measured current was observed as a consequence of the re-adsorption of a trace amount of adsorbates on the sample surfaces with an adsorption time of $74.8 \mathrm{~s}$. When turning the UV light on, a very small increment occurred due to the contribution of photo-generated charge carriers within $\sim 2.0 \mathrm{~s}$, which was followed by an exponential increment in the current due to photo- desorption process with a desorption time of $35.4 \mathrm{~s}$. The adsorption rate with the estimated time constant ( $\tau_{\mathrm{ad}}=74.8 \mathrm{~s}$ ) was found to be smaller than desorption rate $\left(\tau_{\text {des }}=35.4 \mathrm{~s}\right)$ of $\mathrm{B}-\mathrm{ZnO}$ sample. The drastic difference between the adsorption and desorption rates leads to a downward trend in the overall current variation within a couple of on/off cycles as seen in Fig. 5(a). On the other hand, after the $\mathrm{SiO}_{2}$ encapsulation of $\mathrm{ZnO}$ surface, the sharp drop time decreased to $0.8 \mathrm{~s}$ as turning the $\mathrm{UV}$ light off, and the adsorption rate was estimated as $30.6 \mathrm{~s}$. When turning the UV light on, photo-generation occurred within a $0.7 \mathrm{~s}$, which was followed by an exponential increment with a desorption rate of $19.3 \mathrm{~s}$, at the sensitivity limits of our measuring system. These results apparently indicate that the surface encapsulation method notably reduce the estimated time constants for both adsorption and desorption rates. Additionally, it reveals more reasonable photo-generation and recombination time constants. Besides, when the amplitudes of current variations of $\mathrm{B}-\mathrm{ZnO}$ and $\mathrm{E}-\mathrm{ZnO}$ samples were compared, the amplitude of $\mathrm{E}-\mathrm{ZnO}$ sample was almost one order of magnitude smaller than that of bare counterpart, indicating adsorbates responsible for high photoconductivity, and the effect of adsorbate molecules were partially eliminated after encapsulation process.

The photo-response properties of $\mathrm{ZnO}$ thin films greatly improved after the encapsulation of surface with a thin layer of $\mathrm{SiO}_{2}$. The TPS plots of $\mathrm{E}-\mathrm{ZnO}$ thin film exhibit an almost symmetric trend. Once the UV light is turned off, the photo-current of the E-ZnO sample decreased to its initial level indicating enhanced stability and reversibility of our device as seen in Fig. 5(b). Despite the encapsulation significantly improve the device performance, there would be another possible mechanism that still leads to exponential behavior seen in Fig. 5(b), as explained in the discussion section. 


\section{Discussion}

Possible technological applications of $\mathrm{ZnO}$ materials in the fields of gas sensors and optoelectronics have been investigated for many years [36], but the relation between the material surface and atmospheric adsorbates have not been fully understood yet. In fact, its photo-response is mainly governed by the charge transfer doping phenomenon that occur during adsorption/desorption of adsorbates in air as previously discussed [10,37]. When the adsorbates like $\mathrm{O}_{2}$ and $\mathrm{H}_{2} \mathrm{O}$ in air are stuck on the surface of $\mathrm{ZnO}$ nanostructures especially with high surface area-to-volume ratio, they trap free electrons by creating a low conductivity depletion layer in the vicinity of surface. The trapped electrons are released fast at first via desorption of $\mathrm{H}_{2} \mathrm{O}$ molecules, and then continues at a slower rate due to desorption of $\mathrm{O}_{2}$ molecules under UV light that promotes desorption process. As evidenced in our XPS results, the PED-fabricated ZnO thin films possess oxygen vacancies on the surface, which they serve active areas for adsorption of $\mathrm{O}_{2}$ and $\mathrm{H}_{2} \mathrm{O}$ molecules. These results confirm our suggestions about adsorption/desorption mechanism in $\mathrm{ZnO}$ thin films with large surface area-to-volume ratios.

For $\mathrm{ZnO}$ thin film with inherently high surface area-to-volume ratio as a result of high energy deposition technique like PED in our case, the conductivity is mainly governed by the adsorption/desorption of atmospheric adsorbates. In the light of the experimental results obtained in 5 ks photo-response measurements (Fig. 4), the conductivity changes can be written in the form of $\Delta \sigma_{Z n O}(t) \propto \sigma_{\text {des }}(t)$ under UV illumination. Here, $\sigma_{\text {des }}(t)$ is the contribution of photo-desorption of adsorbates from surfaces to conductivity. The contribution of photo-desorption process, $\sigma_{\text {des }}(t)$, can be divided into two components as $\sigma_{\text {des }}(t)=$ $\sigma_{\mathrm{H}_{2} \mathrm{O}}^{f}(t)+\sigma_{\mathrm{O}_{2}}^{S}(t)$. Here, $\sigma_{\mathrm{H}_{2} \mathrm{O}}^{f}(t)$ is the contribution of fast component by reason of water desorption, while $\sigma_{O_{2}}^{S}(t)$ is the contribution of slow component by reason of oxygen desorption. These fast and slow terms vary as a function of time in the form of $\sigma_{\mathrm{H}_{2} \mathrm{O}}^{f}(t)=1-$ $e^{-t / \tau_{f}}$ and $\sigma_{O_{2}}^{S}(t)=1-e^{-t / \tau_{s}}$ corresponding to the first and second terms in the right side of Eq. (2), respectively. On the other hand, upon turning the UV light off the alteration in conductivity occurred as $\Delta \sigma_{Z n o}(t) \propto-\sigma_{a d}(t)$. Here $\sigma_{a d}(t)$ is the contribution of the readsorption of adsorbates on the surface of film. The same two components (fast and slow) aforementioned are responsible for exponential decay of photo-response. Therefore, fast and slow decay processes change as a function of time $\sigma_{H_{2} O}^{f}(t)=e^{-t / \tau_{f}}$ and $\sigma_{O_{2}}^{s}(t)=e^{-t / \tau_{s}}$, respectively. 
To get deeper insights into the photo-response character of $\mathrm{ZnO}$ thin film under UV light, we have conducted short period TPS measurements. The results reveal that the conductivity variations can be written in the form of $\Delta \sigma_{B-Z n o}(t) \propto \sigma_{p h}+\sigma_{d e s}(t)$ under UV exposure, $\sigma_{p h}$ is the contribution of photo-induced charge carriers in the depletion layers of $\mathrm{ZnO}$ thin film. When the UV light is turned off, the conductivity changes can be written as $\Delta \sigma_{B-Z n O}(t) \propto-\left(\sigma_{p h}+\sigma_{d e s}(t)\right)$. The observed exponential trend proves the $\sigma_{p h} \ll$ $\sigma_{\text {des }}(t)$ due to surface layers that absorb most of UV photons for desorption process.

In this work, we showed an experimental method that inhibits the interactions between atmosphere and surface simply by a $\mathrm{SiO}_{2}$ encapsulation layer. Therefore, we assumed that the contribution of adsorbates to the photo-response character was completely blocked by means of encapsulation. However, despite the effect of adsorbates on the photocurrent variation was inhibited, the exponential trend was still observed in Fig. 5(b). As known in literature, the existence of defects in the crystal structure would introduce several defect-induced states acting as hole trap centers within the bandgap of any semiconductor $[14,38,39]$. Also, oxygen vacancies are the predominant type of defect in metal oxides semiconductors such as $\mathrm{In}_{2} \mathrm{O}_{3}$ and ZnO thin films especially produced by PLD or PED techniques [20,40]. Our PL and XPS results confirm the existence of many defective states both on the surface and within the bandgap of PED-fabricated $\mathrm{ZnO}$ thin films. Therefore, even if we isolate the surface of $\mathrm{ZnO}$ thin film from the environment, the photo-generated holes are mostly trapped at these defect states leaving behind unpaired electrons in the $\mathrm{ZnO}$ that still contribute to the photocurrent. Due to the lifetime of these unpaired electrons is further increased by trapped holes; the recombination rate of electron-hole pairs is progressively slowing down. The additional contribution of trap-states to the conductivity $\left(\sigma_{\text {trap }}\right)$ plays another role on the overall conductivity variation of $\mathrm{ZnO}$ thin film $\left(\Delta \sigma \propto \sigma_{p h}+\sigma_{\text {trap }}\right)$ determined by the UV light is onoff. Eventually, ZnO thin film shows slow photo-response to UV light leading to exponential behavior as still seen in Fig. 5(b).

\section{Conclusion}

In summary, we investigated the mechanism behind slow photo-response behavior of the PED-fabricated $\mathrm{ZnO}$ thin films. The photo-response of $\mathrm{ZnO}$ occurs because of two mechanisms acting simultaneously, which make it difficult to pick out the individual contributions since the effect of adsorbates is dominant. To eliminate the effect of adsorbate, we encapsulated the surface of $\mathrm{ZnO}$ with a thin layer of $\mathrm{SiO}_{2}$. We see that defect-induced hole 
trap-states plays another role on the overall conductivity variation of $\mathrm{ZnO}$ thin film, which still leads to slow photo-response to continue. Therefore, controlling intrinsic defects during material growth process as well as encapsulation process is important to obtain fast speed UV photodetector fabricated out of $\mathrm{ZnO}$ material.

We believe that our results lead the way for future application of high speed and sensitive $\mathrm{ZnO}$ photodetector with choosing effective encapsulation materials and prompt for further investigation in encapsulation of other n-type materials.

\section{Acknowledgments}

The authors would like to thank Serap Yiğen for the encapsulation experiments, and S. Batuhan Kalkan for the experimental setup. Characterizations of samples were conducted in the Center for Materials Research of İzmir Institute of Technology (IYTE-MAM) and Ege University Central Research Test \& Analysis Laboratory Application \& Research Center (EGE-MATAL). A part of this work has been supported by Ege University Scientific Research Project (BAP) with Project No. 15-FEN-058.

\section{References}

[1] Özgür Ü, Alivov Y I, Liu C, Teke A, Reshchikov M A, Doğan S, Avrutin V, Cho S-J and Morkoç H 2005 A comprehensive review of ZnO materials and devices J. Appl. Phys. 98041301

[2] Ghosh M, Ningthoujam R S, Vatsa R K, Das D, Nataraju V, Gadkari S C, Gupta S K and Bahadur D 2011 Role of ambient air on photoluminescence and electrical conductivity of assembly of ZnO nanoparticles J. Appl. Phys. 110054309

[3] Claflin B, Look D C and Norton D R 2007 Changes in Electrical Characteristics of ZnO Thin Films Due to Environmental Factors J. Electron. Mater. 36 442-5

[4] Bhatt V, Kumar M, Kim J, Chung H-J and Yun J-H 2019 Persistent photoconductivity in Al-doped ZnO photoconductors under air, nitrogen and oxygen ambiance: Role of oxygen vacancies induced DX centers Ceram. Int. 45 8561-70

[5] Zhu L and Zeng W 2017 Room-temperature gas sensing of ZnO-based gas sensor: A review Sensors Actuators A Phys. 267 242-61

[6] Soci C, Zhang A, Xiang B, Dayeh S A, Aplin D P R, Park J, Bao X Y, Lo Y H and Wang D 2007 ZnO Nanowire UV Photodetectors with High Internal Gain Nano Lett. 7 1003-9

[7] Han P, Adler E R, Liu Y, St Marie L, El Fatimy A, Melis S, Van Keuren E and Barbara P 2019 Ambient effects on photogating in MoS 2 photodetectors Nanotechnology 30284004

[8] Chang H-C, Huang Y-J, Chang H-Y, Su W-J, Shih Y-T, Huang Y-S and Lee K-Y 2014 Oxygen 
adsorption effect on nitrogen-doped graphene electrical properties Appl. Phys. Express 7055101

[9] Ahn J-H, Parkin W M, Naylor C H, Johnson A T C and Drndić M 2017 Ambient effects on electrical characteristics of CVD-grown monolayer MoS2 field-effect transistors Sci. Rep. 74075

[10] Li Y, Della Valle F, Simonnet M, Yamada I and Delaunay J-J 2009 Competitive surface effects of oxygen and water on UV photoresponse of ZnO nanowires Appl. Phys. Lett. 94023110

[11] Jin Y, Wang J, Sun B, Blakesley J C and Greenham N C 2008 Solution-Processed Ultraviolet Photodetectors Based on Colloidal ZnO Nanoparticles Nano Lett. 8 1649-53

[12] Zhou J, Gu Y, Hu Y, Mai W, Yeh P-H, Bao G, Sood A K, Polla D L and Wang Z L 2009 Gigantic enhancement in response and reset time of ZnO UV nanosensor by utilizing Schottky contact and surface functionalization Appl. Phys. Lett. 94191103

[13] Li Q H, Gao T, Wang Y G and Wang T H 2005 Adsorption and desorption of oxygen probed from ZnO nanowire films by photocurrent measurements Appl. Phys. Lett. 86123117

[14] Moazzami K, Murphy T E, Phillips J D, Cheung M C K and Cartwright A N 2006 Sub-bandgap photoconductivity in $\mathrm{ZnO}$ epilayers and extraction of trap density spectra Semicond. Sci. Technol. 21 $717-23$

[15] Ahn S-E, Ji H J, Kim K, Kim G T, Bae C H, Park S M, Kim Y-K and Ha J S 2007 Origin of the slow photoresponse in an individual sol-gel synthesized ZnO nanowire Appl. Phys. Lett. 90153106

[16] Özdoğan M, Yiğen S, Çelebi C and Utlu G 2019 The comparison of transient photocurrent spectroscopy measurements of Pulsed Electron Deposited ZnO thin film for air and vacuum ambient conditions Thin Solid Films 680 48-54

[17] Kufer D and Konstantatos G 2015 Highly Sensitive, Encapsulated MoS 2 Photodetector with Gate Controllable Gain and Speed Nano Lett. 15 7307-13

[18] Park W, Park J, Jang J, Lee H, Jeong H, Cho K, Hong S and Lee T 2013 Oxygen environmental and passivation effects on molybdenum disulfide field effect transistors Nanotechnology 24095202

[19] Kalkan S B, Aydın H, Özkendir D and Çelebi C 2018 The effect of adsorbates on the electrical stability of graphene studied by transient photocurrent spectroscopy Appl. Phys. Lett. 112013103

[20] Tricot S, Nistor M, Millon E, Boulmer-Leborgne C, Mandache N B, Perrière J and Seiler W 2010 Epitaxial ZnO thin films grown by pulsed electron beam deposition Surf. Sci. 604 2024-30

[21] Ali A, Henda R and Fagerberg R 2017 Effect of temperature and discharge voltage on the properties of Co-doped ZnO thin films deposited by pulsed electron beam ablation Appl. Surf. Sci. 422 1082-92

[22] Nistor M, Seiler W, Hebert C, Matei E and Perrière J 2014 Effects of substrate and ambient gas on epitaxial growth indium oxide thin films Appl. Surf. Sci. 307 455-60

[23] Nistor M, Mandache N B and Perrière J 2008 Pulsed electron beam deposition of oxides thin films $J$. 
Phys. D. Appl. Phys. 41165205

[24] Naouar M, Ka I, Gaidi M, Alawadhi H, Bessais B and Khakani M A E 2014 Growth, structural and optoelectronic properties tuning of nitrogen-doped $\mathrm{ZnO}$ thin films synthesized by means of reactive pulsed laser deposition Mater. Res. Bull. 57 47-51

[25] Meng L-J, Moreira de Sá C P and dos Santos M P 1994 Study of the structural properties of ZnO thin films by x-ray photoelectron spectroscopy Appl. Surf. Sci. 78 57-61

[26] Hsieh P-T, Chen Y-C, Kao K-S and Wang C-M 2007 Luminescence mechanism of ZnO thin film investigated by XPS measurement Appl. Phys. A 90 317-21

[27] Lu Y F, Ni H Q, Mai Z H and Ren Z M 2000 The effects of thermal annealing on ZnO thin films grown by pulsed laser deposition J. Appl. Phys. 88 498-502

[28] Chen M, Wang X, Yu Y ., Pei Z ., Bai X ., Sun C, Huang R . and Wen L . 2000 X-ray photoelectron spectroscopy and auger electron spectroscopy studies of Al-doped ZnO films Appl. Surf. Sci. 158 134-40

[29] Khokhra R, Bharti B, Lee H-N and Kumar R 2017 Visible and UV photo-detection in ZnO nanostructured thin films via simple tuning of solution method Sci. Rep. 715032

[30] Mandal S, Goswami M L N, Das K, Dhar A and Ray S K 2008 Temperature dependent photoluminescence characteristics of nanocrystalline ZnO films grown by sol-gel technique Thin Solid Films 516 8702-6

[31] Lim K, Abdul Hamid M, Shamsudin R, Al-Hardan N H, Mansor I and Chiu W 2016 TemperatureDriven Structural and Morphological Evolution of Zinc Oxide Nano-Coalesced Microstructures and Its Defect-Related Photoluminescence Properties Materials (Basel). 9300

[32] Ahn C H, Kim Y Y, Kim D C, Mohanta S K and Cho H K 2009 A comparative analysis of deep level emission in ZnO layers deposited by various methods J. Appl. Phys. 105013502

[33] Bandopadhyay K and Mitra J 2015 Zn interstitials and O vacancies responsible for n-type ZnO: what do the emission spectra reveal? RSC Adv. 5 23540-7

[34] Vanheusden K, Warren W L, Seager C H, Tallant D R, Voigt J A and Gnade B E 1996 Mechanisms behind green photoluminescence in ZnO phosphor powders J. Appl. Phys. 79 7983-90

[35] Panda S K and Jacob C 2012 Preparation of transparent ZnO thin films and their application in UV sensor devices Solid. State. Electron. 73 44-50

[36] Liu K, Sakurai M and Aono M 2010 ZnO-Based Ultraviolet Photodetectors Sensors 10 8604-34

[37] Keem K, Kim H, Kim G-T, Lee J S, Min B, Cho K, Sung M-Y and Kim S 2004 Photocurrent in ZnO nanowires grown from Au electrodes Appl. Phys. Lett. 84 4376-8

[38] Jiang J, Ling C, Xu T, Wang W, Niu X, Zafar A, Yan Z, Wang X, You Y, Sun L, Lu J, Wang J and Ni Z 2018 Defect Engineering for Modulating the Trap States in 2D Photoconductors Adv. Mater. 301804332 
[39] Fei-Fei W, Chong W, Ke C and Bing-Suo Z 2008 Trap induced slow photoresponse of single CdS nanoribbons Chinese Phys. B 17 3103-7

[40] Seiler W, Nistor M, Hebert C and Perrière J 2013 Epitaxial undoped indium oxide thin films: Structural and physical properties Sol. Energy Mater. Sol. Cells 116 34-42 